\title{
Companion Paper for "MiniView Layout for Bandwidth-Efficient 360-Degree Video"
}

\author{
Mengbai Xiao \\ The Ohio State University \\ xiao.736@osu.edu \\ Li Liu \\ George Mason University \\ lliu8@gmu.edu \\ Songqing Chen \\ George Mason University \\ sqchen@gmu.edu
}

\author{
Shuoqian Wang \\ SUNY Binghamton \\ swang130@binghamton.edu \\ Zhenhua Li \\ Tsinghua University \\ lizhenhua1983@tsinghua.edu.cn \\ Lucile Sassatelli \\ Universite Cote d'Azur, CNRS, I3S \\ sassatelli@i3s.unice.fr
}

\author{
Chao Zhou \\ SUNY Binghamton \\ czhou5@binghamton.edu \\ Yao Liu \\ SUNY Binghamton \\ yaoliu@binghamton.edu \\ Gwendal Simon \\ IMT Atlantique \\ gwendal.simon@imt-atlantique.fr
}

\begin{abstract}
This artifact includes source code, scripts and datasets required to reproduce the experimental figures in the evaluation of the MM'18 paper, which is entitled "MiniView Layout for Bandwidth-Efficient 360-Degree Video" [3]. The artifact reports the comparison results among the standard cube layout (CUBE), the equi-angular layout (EAC), and the MiniView layout (MVL) in terms of compressed video size, visual quality of views and decoding and rendering time.
\end{abstract}

\section{CCS CONCEPTS}

- Information systems $\rightarrow$ Multimedia content creation.

\section{KEYWORDS}

360 video, virtual reality, view-adaptive encoding

\section{ACM Reference Format:}

Mengbai Xiao, Shuoqian Wang, Chao Zhou, Li Liu, Zhenhua Li, Yao Liu, Songqing Chen, Lucile Sassatelli, and Gwendal Simon. 2019. Companion Paper for "MiniView Layout for Bandwidth-Efficient 360-Degree Video". In Proceedings of the 27th ACM International Conference on Multimedia (MM '19), October 21-25, 2019, Nice, France. ACM, New York, NY, USA, 4 pages. https://doi.org/10.1145/3343031.3351168

\section{ARTIFACT DESCRIPTION}

The artifact is available at

https://github.com/bingsyslab/mm19-artifact

without the datasets used in the evaluation. The datasets are accessible at

https://dl. dropboxusercontent. com/s/snlomfjoh7ybsk3/dataset. tgz , and it can also be automatically downloaded by running the script in the artifact. The artifact includes a patch to ffmpeg (360-project

Permission to make digital or hard copies of all or part of this work for personal or classroom use is granted without fee provided that copies are not made or distributed for profit or commercial advantage and that copies bear this notice and the full citation on the first page. Copyrights for components of this work owned by others than ACM must be honored. Abstracting with credit is permitted. To copy otherwise, or republish, to post on servers or to redistribute to lists, requires prior specific permission and/or a fee. Request permissions from permissions@acm.org.

MM '19, October 21-25, 2019, Nice, France

(C) 2019 Association for Computing Machinery.

ACM ISBN 978-1-4503-6889-6/19/10 . \$ \$15.00

https://doi.org/10.1145/3343031.3351168 filter), running scripts, documentations and a small dataset for verification.

\subsection{Folder structure}

The folder structure of the artifact is shown as follows:

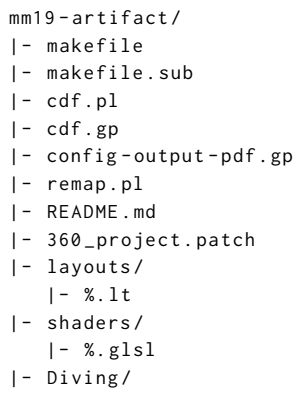

makefile: the script controlling experimental setups, evaluations, and plotting.

makefile. sub: the script carrying out evaluations for one video. cdf.pl: the script collecting metrics from a set of videos.

config-output-pdf.gp, cdf.gp: the scripts plotting CDF figures. remap.pl: the script producing 360-degree videos with a specific layout from an HD equirectangular video.

README.md: the documentation of the artifact.

360_project. patch: the patch file to ffmpeg that installs the 360project filter.

layouts/: the folder holding different layout files.

shaders/: the folder holding relevant OpenGL shaders.

Diving/: the example video dataset.

\subsection{Datasets}

The videos and user traces are from publicly available datasets $[1,2]$. For fairness, we transcode all videos into the equirectangular layout at the resolution of $3840 \times 2048$, and these videos are chunked into segments of 1 second. In addition, the user orientation traces are unified into . txt files, in which a line is a sample and it looks like

$0.1410334 .000000-15.787280174 .084166$

. The items are the timestamp in seconds, the line number, and the head rotations surrounding $\mathrm{x}$-axis and $\mathrm{y}$-axis, respectively. 
Table 1: Dataset description

\begin{tabular}{lll}
\hline & \# of traces & Video names \\
\hline Dataset-1 [1] & 53 & Diving, Paris, Rollercoaster, Timelapse, Venise \\
Dataset-2 [2] & 48 & Conan_Gore_Fly, Cooking_Battle, Front, Help, Rhinos, \\
& & Conan_Weird_Al, Football, Tahiti_Surf, FemaleBasketball, \\
& & Fighting, Korean, Reloaded, RioVR, TFBoy, VoiceToy, Anitta \\
\hline
\end{tabular}

We ignore the head rotation surrounding $\mathrm{z}$-axis since users rarely change their head around this axis. The video segments and the orientation files are named in the formats of $\$\{\mathrm{sec}\} \_s e c . m p 4$ and uid-\$\{uid\}_raw.txt, respectively.

The datasets include 21 videos. 5 of them have 58 users' view orientations while the other 16 videos have 48 users' head movement traces. The list of these video names are shown in Table 1 . In the evaluation, we distinguish them into two groups: moving-camera and static-camera videos. In Table 1, the moving-camera videos are presented in bold.

\section{INSTALLATION}

Before the experiment workflow, an ffmpeg with 360-project filter need to be built and all datasets should be downloaded.

\subsection{Dependencies}

The 360-project filter has been tested with the master branch of ffmpeg with the ref

$$
37 \text { e4c226c06c4ac6b8e3a0ccb2c0933397d6f96f }
$$

and is expected to be correctly built with the latest ffmpeg source code. The experiments are successfully carried out on Ubuntu 18.04 and Ubuntu 16.04. The tested OpenGL version is OpenGL 3.3 (Core Profile) Mesa 18.0.5. The scripts in the artifact use Gnuplot 5.2 patchlevel 2, Perl 5.018 and GNU Make 4.1.

To install the software dependencies on a Ubuntu system, run the following commands:

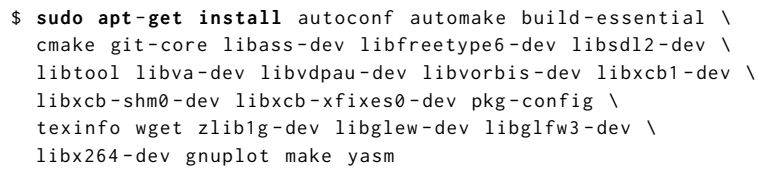

Hardware dependencies: Running this artifact on a platform equipped with a GPU is highly recommended, which can greatly accelerate the projection operations.

\subsection{Running artifact over ssh}

Since the artifact is developed based on GLFW, it requires a display to create a window, though it is invisible. For running this artifact on a server without a monitor connected via ssh, the virtual framebuffer needs to be installed:

| \$ sudo apt-get install xvfb

In the experiment workflow, two commands ("make remap-videos" and "make view-videos") processing videos with OpenGL need to be prefixed with svfb-run to ensure their successful run. For example:
| \$ xvfb-run make view-videos VNAMES=Diving

Details of these two commands are discussed in Section 3.1.

\subsection{Artifact installation}

(1) Download the artifact with the following instructions:

| \$ git clone https://github.com/bingsyslab/mm19-artifact

(2) Download, patch, and build ffmpeg with 360-project filter.

- Use the makefile script to automatically download, patch, and build ffmpeg:

\$ cd mm19-artifact

\$ make prepare-ffmpeg

If $f f m p e g$ has been successfully built, two binaries $f f m p e g / f f m p e g$

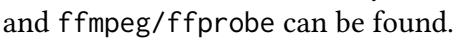

- If manually building ffmpeg is preferred, follow the instructions below:

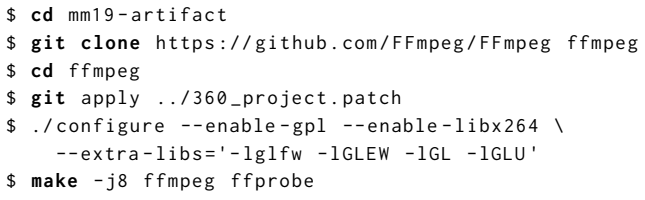

(3) To download the datasets:

| \$ make prepare-dataset

This will automatically download a tarball of the dataset named dataset.tgz and extract it into a list of folders. The tarball is $6.9 \mathrm{G}$ and the dataset takes additional $7.6 \mathrm{G}$ disk space after decompression. The tarball can also be accessed via the link shown in Section 1. A video to be tested is organized in the format:

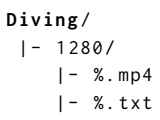

For a video "Diving", it should contain the HD equirectangular 360-degree video segments and the orientation files. Both of them are stored in the directory 1280/. Following the details in Section 1.2 helps add a new test video into the experiment.

\section{EXPERIMENT WORKFLOW}

The experiment follows three major steps: 1) 360-degree video generation, 2) view video generation, and 3) experimental measurements. A full run of this artifact on the provided 21 videos (with the default configurations) takes $\sim 12$ hours, and an additional $\sim 80 \mathrm{G}$ disk space will be occupied. The workflow can also be carried out on a single video for proving its completeness. In this section, we 
Table 2: Experimental parameters that can be customized

\begin{tabular}{|c|c|c|c|c|}
\hline Name & Description & Value format & Default value & Value as a vector? \\
\hline MOVING_VNAMES & The names of videos as moving-camera type & $\{$ Diving, Paris, ... $\}$ & $\begin{array}{l}\text { Diving, Rollercoaster, } \\
\text { Tahiti_Surf, Front }\end{array}$ & Yes \\
\hline LAYOUTS & The names of tested layouts & $\{$ cube, eac, mv $\}$ & cube eac mv & Yes \\
\hline SCHEMES & The compression parameters passed to $\times 264$ & $\{\operatorname{crf} \$\{N\}\}$ & $\operatorname{crf23}$ & Yes \\
\hline CUBE_RES & The resolution of CUBE layout & $\$\{R E S\} \times \$\{R E S\}$ & $1920 \times 1280$ & No \\
\hline EAC_RES & The resolution of EAC layout & $\$\{\mathrm{RES}\} \times \$\{\mathrm{RES}\}$ & $1920 \times 1280$ & $\mathrm{No}$ \\
\hline MV_RES & The resolution of MiniView layout & $\$\{R E S\} \times \$\{R E S\}$ & $2240 \times 832$ & No \\
\hline FOV & The FoV of generated view videos & $\$\{$ DEGREE $\} \times \$\{$ DEGREE $\}$ & $100 \times 100$ & No \\
\hline
\end{tabular}

will first present how to carry out the experiment on an example video "Diving". Then we have an integrated command to run the full experiment presented in our original paper. We will further show how to customize this artifact.

\subsection{Video generation}

(1) To generate 360-degree videos for a given video:

\$ make remap-videos VNAMES=Diving

This command generates different layouts of 360-degree videos, where the option VNAMES specifies which video is used. This artifact is extensively customizable and more details can be found in Section 3.4. The output videos are generated into crf23/remaps/ in the names of $\$\{$ layout $\} \$\{$ time $\}$. mp4.

(2) To generate view videos:

| \$ make view-videos VNAMES=Diving

The view videos directly generated from the HD equirectangular videos (1280/\$ time $\}$.mp4) are named as \%.hd.mp4 in views/ while the view videos generated from different layouts of 360degree videos are located at crf23/views/.

\subsection{Video analysis}

(3) To measure various metrics:

\$ make psnr-logs VNAMES=Diving
$\$$ make ssim-logs VNAMES=Diving

$\$$ make ts-logs VNAMES=Diving

These commands measure PSNR, SSIM as well as rendering and decoding time, which are preserved as log files stored at $\mathrm{crf} 23 / \mathrm{psnr} /, \mathrm{crf} 23 / \mathrm{ssim} /$, and $\mathrm{crf} 23 / \mathrm{ts} /$, respectively. With the videos and $\log$ files generated, a test video folder looks like:

$$
\begin{aligned}
& \text { |- Diving/ } \\
& \mid-1280 / \\
& \mid-\$\{\text { time }\} . m p 4 \\
& \mid- \text { uid- } \$\{\text { uid }\} \text { raw.txt } \\
& \mid- \text { crf23/ } \\
& \mid- \text { remaps/ } \\
& \mid-\$\{\text { layout }\} \$\{\text { time }\} . m p 4
\end{aligned}
$$

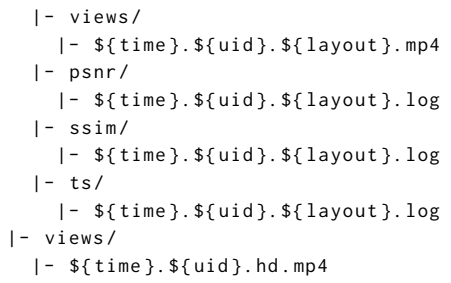

(4) Generate the figures in the PDF format:

\$ make cdfs pdfs VNAMES=Diving

Two types of files are generated with this command. \%.cdf is the data file and \%. pdf files are the figures. There will be four figures showing the measured PSNR (psnr_avg.crf23-psnr . pdf), SSIM (All.crf23-ssim.pdf), video size (size.crf23-remaps.pdf) and rendering time (ts.crf23-ts.pdf).

\subsection{Re-run the full experiment}

To repeat the experiments presented in our original paper, use the following command

| \$ make psnr-ssim-ts-rand moving-static-cdfs moving-static-pdfs

The configuration of this experiment is the default ones shown in Section 3.4. It worth noting that this will generate 7 figures since we distinguish the 21 videos into moving-camera videos and static-camera videos for PSNR, SSIM and video size measurement.

\subsection{Experiment customization}

The experiment can be extensively customized. The comprehensive customizable parameters are listed in Table 2. An example of customized experiment can be launched like

\$ make psnr-ssim-ts-rand VNAMES=Paris $\mathrm{FOV}=90 \times 90$ LAYOUTS $=\mathrm{mV}$ SCHEMES $=c r f 18$ UID_NR $=1$ TIMES_NR $=5$

This means we only carry experiments over the Paris video and only test the MiniView layout. When generating the MiniView videos, the compression parameter uses $c r f=18$. The FoV of view 
videos is $90^{\circ} \times 90^{\circ}$. We randomly test over 5 video segments and 1 orientation trace.

\section{360-PROJECT FILTER}

We implement 360-project as an ffmpeg filter that generates a user view from an input 360-degree frame. Understanding this tool is not necessary to evaluate the MiniView Layout. We will give a brief description to this tool and further details can be found in the documentation of this artifact.

An example of using this filter is like:

$\$$./ffmpeg -loglevel 'info' -y -i cube.mp4 -filter:v

"project $=800: 800: 90: 90: 0: 180: 0:$ vertex.glsl:eqdis.glsl: $:$ cube. $1 \mathrm{t}: 0.0: 1 . \theta^{\prime \prime}$ । back. mp 4

This command generates a view video from the 360-degree video cube.mp4. The output view video has the resolution of $800 \times 800$, and its $\mathrm{FoV}$ is $90^{\circ} \times 90^{\circ}$. The video shows what a user will watch as she turns her head following $\left\{0^{\circ}, 180^{\circ}, 0^{\circ}\right\}$, which is exactly the view from her back. An orientation file can be passed in to simulate the user's head movement. vertex.glsl and eqdis.glsl are the vertex shader and the fragment shader used to produce the view video. cube. $1 \mathrm{t}$ is the layout file describing the input video.

\section{MISCELLANEOUS REMARKS}

- In the artifact, a list of commands can be used to ease clean operations:

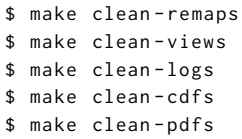

These commands remove remapped videos, view videos, experimental logs, CDF data files, and PDF figures, respectively.

- If you prefer building the ffmpeg at another place, you need to redirect the ffmpeg folder in the makefile:

export FFMPEG_DIR := $\$\{$ PATH_TO_FFMPEG $\}$

\section{NOTES FROM REVIEWERS}

Two co-authors of this paper are reviewers of this companion paper. We have successfully installed the library and reproduced the results that were initially presented in the original paper [3]. We also acknowledge the efforts of the authors of the original article to make the reproducibility process as smooth as possible. We would like in particular to emphasize two key improvements that have been implemented during the reviewing process to make the code source easier to manipulate for other researchers.

First, the initial code was presented such as it was tempting to manipulate the whole package as a "black box." One unique command was supposed to launch the full process of preparing the video, testing the quality, collecting the data, and plotting the figures. It was still possible to dive into the code source if one wanted to make the process step by step, but the main make commands did not allow a fraction of the whole processing. We believe that other researchers interested in testing the new layouts are interested by implementing either only the video processing part (typically to build the miniview videos and to run other types of evaluation on this layout), or by implementing only the evaluation part (typically to test the results of their own projection against miniview layout in the same performance evaluation configuration). As a result of this observation, and after a redefinition of the script commands, the original unique command has been split into multiple commands, which provide more flexibility for researchers in the area.

Second, we were initially not able to run the code properly due to the ssh issue explained in Section 2.2. We were at this time puzzled: on the one hand our mission was essentially to reproduce the results, in the sense that our primary goal was to check whether the scientific results are true. Even though it is more comfortable to set a machine with the right configuration from remote public datacenters, this comfort is not a requirement to assess that the results are valid. On the other hand, the code that is presented in the paper is supposed to run in a server, potentially many servers in a content delivery system. These machines do not have a display monitor and can only be accessed remotely. It is our understanding that one of the motivations for sharing code is to ease the development of prototypes based on the proposal by third-party researchers. It was clear that this objective cannot be fulfilled without ssh-friendly implementation. Fortunately the original authors proposed some fixes, which not only allow us to run the code as in the original paper in a comfortable way, but also allow third-party researchers to implement part of this code in a prototype.

Overall we have been able to reproduce the initial experiments with similar results as what was presented in the original paper. The code now enables running a fraction of the code, hopefully to the benefit of other researchers, and it can run in any data-center, with respect to the hardware and software requirements.

\section{ACKNOWLEDGMENTS}

This work is partially supported by NSF under grants CNS-1524462 and CNS-1618931.

\section{REFERENCES}

[1] Xavier Corbillon, Francesca De Simone, and Gwendal Simon. 2017. 360-Degree Video Head Movement Dataset. In Proceedings of the 8th ACM on Multimedia Systems Conference (MMSys'17). ACM, 199-204.

[2] Chenglei Wu, Zhihao Tan, Zhi Wang, and Shiqiang Yang. 2017. A Dataset for Exploring User Behaviors in VR Spherical Video Streaming. In Proceedings of the 8th ACM on Multimedia Systems Conference (MMSys'17). ACM, 193-198.

[3] Mengbai Xiao, Shuoqian Wang, Chao Zhou, Li Liu, Zhenhua Li, Yao Liu, and Songqing Chen. 2018. MiniView Layout for Bandwidth-Efficient 360-Degree Video. In Proceedings of the 26th ACM International Conference on Multimedia (MM '18). ACM, 914-922. 